\title{
基于新型萗环稠合嗍氟二吡咯化合物(BODIPY)的氟离子 荧光探针及其细胞成像研究
}

\author{
周建平 $a$ 吴保庚 ${ }^{a}$ 周志宽 $*, a$ 田蒋为 $*, b$ 袁爱华 $*, a$ \\ ( ${ }^{a}$ 江苏科技大学环境与化学工程学院 镇江 212003) \\ ( ${ }^{b}$ 中国药科大学中药学院 南京 211198)
}

\begin{abstract}
摘要 设计、合成了一种基于新型荎环稠合嗍氟二吡咯化合物(BODIPY) 5 的氟离子比率计量型和苂光猝灭型分子探 针. 通过紫外-可见光谱实验发现，该探针在氟离子存在时光谱红移 $100 \mathrm{~nm}$, 进入近红外区域，可用于肉眼比色检测. 荧光光谱分析表明，氟离子可促使苂光猝灭. 细胞成像研究表明探针分子 $\mathbf{5}$ 可在活体细胞中专一性地识别氟离子. 关键词 嗍氟二吡咯化合物(BODIPY); 近红外; 氟离子; 细胞成像
\end{abstract}

\section{A Novel Naphthalene-Fused Boron Dipyrromethene (BODIPY)- Based Near Infrared Fluorescent Probe for Detecting Fluoride in Living Cells}

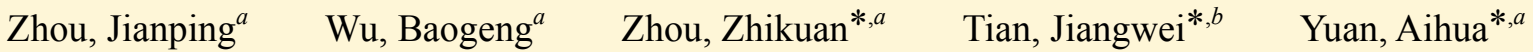 \\ ( ${ }^{a}$ School of Environmental and Chemical Engineering, Jiangsu University of Science and Technology, Zhenjiang 212003) \\ ( ${ }^{b}$ Department of Traditional Chinese Medicine, China Pharmaceutical University, Nanjing 211198)
}

\begin{abstract}
A colorimetric and fluorescent turn-off chemosensor for fluoride based on novel naphthalene-fused boron dipyrromethene (BODIPY) 5 was designed and synthesized. In various anions, only upon addition of fluoride, the UV-Vis absorption peak of probe 5 red-shifted $100 \mathrm{~nm}$, reaching the near infrared (NIR) region. Thus, it can be used as naked-eye detector for fluoride ion. In the fluorescence test, upon addition of fluoride, the fluorescence of probe $\mathbf{5}$ quenched significantly. Confocal fluorescence microscopy experiments demonstrate that $\mathbf{5}$ can be used for monitoring fluoride in living cells.
\end{abstract}

Keywords boron dipyrromethene (BODIPY); near infrared (NIR); fluoride; fluorescent sensor

近年来, 生物体内微环境、离子和小分子的苂光识 别受到了研究者的广泛关注, 因为这些物质在生命体内 发挥着不可或缺的作用 ${ }^{[1]}$. 在各种阴离子中, 氟离子具 有最高的电荷密度、最小的离子半径和强路易斯碱性, 是最重要的微量元素之一. 它与人类健康和环境问题密 切相关 ${ }^{[2]}$, 环境中的氟很容易通过水进入到人体中, 适 量的氟可以治疗骨质疏松和牙齿疾病, 而过量的氟可能 导致急性胃和肾脏疾病 ${ }^{[3]}$ 、氟骨病 ${ }^{[4]}$ 等. 因此对氟离子的 检测和追踪就显得尤为重要 ${ }^{[5]}$.

近红外分子探针 ${ }^{[6]}$ 作为荧光分析法的重要工具之 一, 越来越多地被用于生物体系中离子和小分子的检
测 ${ }^{[7]}$. 近红外 $(>650 \mathrm{~nm})$ 区域的光在传播过程中受到的 干扰小, 对物质透射性高, 光能量低, 在透过生物细胞 时对其损伤较低; 而且在此光谱区, 生物体里小分子自 身的吸收和发射最小, 可以避免生物体散射光以及自苂 光对检测造成影响 ${ }^{[1 a, 8]}$.

近年来，基于氟离子与识别基团的共价或非共价作 用，研究者们设计合成了众多的氟离子探针，然而吸收 和发射在近红外区域的氟离子探针仍然比较少见 ${ }^{[9]}$. 硼 氟二吡咯化合物(Boron Dipyrromethene, BODIPY)因其 具有良好的光谱性质而被广泛关注，如苂光量子产率 高、摩尔吸收系数大、光稳定性好、结构易于修饰等优

\footnotetext{
* Corresponding authors. E-mail: zkzhou@just.edu.cn; jwtian@cpu.edu.cn; aihua.yuan@just.edu.cn Received April 25, 2018; revised July 17, 2018; published online September 11, 2018.

Project supported by the National Natural Science Foundation of China (Nos. 21501073, 21775166, 51672114) and the China Postdoctoral Science Foundation (No. 2017M621662)

国家自然科学基金(Nos. 21501073, 21775166, 51672114)及中国博士后基金(No. 2017M621662)资助项目.
} 
点 ${ }^{[10]}$. BODIPY 的可修饰性强, 通过适当的化学修饰可 以设计一系列具有近红外吸收和发射的荧光染料 ${ }^{[11]}$.

近红外 BODIPY 苂光染料的合成方法有很多种, 其 中最常用的方法之一是通过使用苯环 ${ }^{[12]} 、$ 菲 ${ }^{[13]}$ 、噻吩 ${ }^{[14]}$ 、 苯并噻吩 ${ }^{[15]}$ 等杂环或者芳环与 BODIPY 母核稠合, 使其 共轭体系扩大, 紫外吸收和发光光谱红移. 目前文献报 道了少量基于芳环稠合 BDOIPY 的氟离子苂光探针 ${ }^{[16]}$. 芳环稠合可以使 BODIPY 结构刚性增加, 减少分子内基 团转动引发的能量损失, 从而增强苂光 ${ }^{[17]}$. 然而芳环稠 合 BODIPY 的合成较为困难. 我们设计了一种菜环稠合 BODIPY 的合成方法, 通过理性选择原料, 在一步反应 中实现了 Suzuki-Miyaura-Knoevenagel 的偶联缩合反应, 得到稠环 BODIPY ${ }^{[18]}$. 反应方法简单高效, 合成的不对 称萗环稠合 BODIPY 的 3-位甲基可进一步修饰, 引入离 子检测基团，得到近红外的苂光分子探针.

以开发近红外苂光氟离子探针为目标, 选取新型菜 环稠合 BODIPY 为发光体, 设计了一个比率计量型和荧 光猝灭型探针. 探针分子的合成路线如 Scheme 1 所示, 以 2-位碘代 BODIPY 和(2-甲酰基)苯硼酸为原料, 合成 荎环稠合 BODIPY 3, 菜环的稠合扩展了 BODIPY 的 $\pi$ 共轭体系, 使其光谱发生红移. 在此基础上, 本工作继 续对不对称的荎环稠合 BODIPY 3 进行修饰, 利用 Knoevenagel 缩合反应在其 3-位引入含有 $\mathrm{Si}-\mathrm{O}$ 键的离 子识别基团, 合成了一种新型不对称菜环稠合 BODIPY 探针分子 5. 利用氟离子对 $\mathrm{Si}-\mathrm{O}$ 键的特异性断裂能力, 使探针分子 5 发生结构变化, 产生酚氧离子, 从而使其 光谱发生红移, 分子内电荷转移的产生使苂光猝灭. 我
们对探针结构的变化做了详细的分析, 并尝试将其用于 细胞中氟离子的检测.

\section{1 结果与讨论}

\section{1 探针分子 5 的阴离子识别性能研究}

在乙腈溶液中，探针分子 $\mathbf{5}$ 表现出 BODIPY 特有的 吸收峰型, 在 $615 \mathrm{~nm}$ 处表现出强而尖锐的紫外吸收峰, 对应于 $\mathrm{S}_{0} \rightarrow \mathrm{S}_{1}$ 的跃迁，同时伴随着一个 $580 \mathrm{~nm}$ 的肩峰. 为了考察探针分子 $\mathbf{5}$ 在溶液状态下对不同阴离子的响应 性，我们将其溶解于乙腈中，并配制成 $1.0 \times 10^{-5} \mathrm{~mol} / \mathrm{L}$ 的溶液, 向其分别加入 300 equiv. (即 $3.0 \times 10^{-3} \mathrm{~mol} / \mathrm{L}$ ) 阴 离子的乙腈溶液 $\left(\mathrm{Cl}^{-}, \mathrm{Br}^{-}, \mathrm{F}^{-}, \mathrm{I}^{-}, \mathrm{PO}_{4}^{3-}, \mathrm{HSO}_{3}^{-}, \mathrm{NO}_{2}^{-}\right.$, $\mathrm{SO}_{4}^{2-}, \mathrm{CO}_{3}^{2-}, \mathrm{NO}_{3}^{-}$), 通过紫外-可见光谱进行检测(图 1). 结果表明: 相同条件下, 在加入除 $\mathrm{F}^{-}$以外的其它离 子时, 探针分子 $\mathbf{5}$ 的紫外吸收出峰位置不变, 只是强度 发生了极少的变化. 而当加入 $\mathrm{F}^{-}$时, $615 \mathrm{~nm}$ 附近强的吸 收峰和 $358 \mathrm{~nm}$ 附近较弱的吸收峰分别红移至 716 和 404 $\mathrm{nm}$, 主吸收峰红移了 $101 \mathrm{~nm}$, 进入近红外区域, 强度较 之前减小, 峰型变宽(图 1A), 这使得探针 $\mathbf{5}$ 可用于肉眼 检测 $\mathrm{F}^{-}$. 加入不同浓度氟化四丁基铵(TBAF)溶液，如 图 1B 所示, 随着 $\mathrm{F}^{-}$浓度的增加, $615 \mathrm{~nm}$ 处的吸收峰逐 渐消失, $716 \mathrm{~nm}$ 处的吸收峰强度逐渐增加, 交点出现在 $635 \mathrm{~nm}$, 可用于氟离子的定量分析.

探针分子 5 在乙腈溶液中表现出强而宽的发射峰, 其最大发射波长在 $648 \mathrm{~nm}$. 我们对探针 5 的荧光检测性 能进行了研究. 从图 $2 \mathrm{~A}$ 可以看出, 当加入除 $\mathrm{F}^{-}$之外的
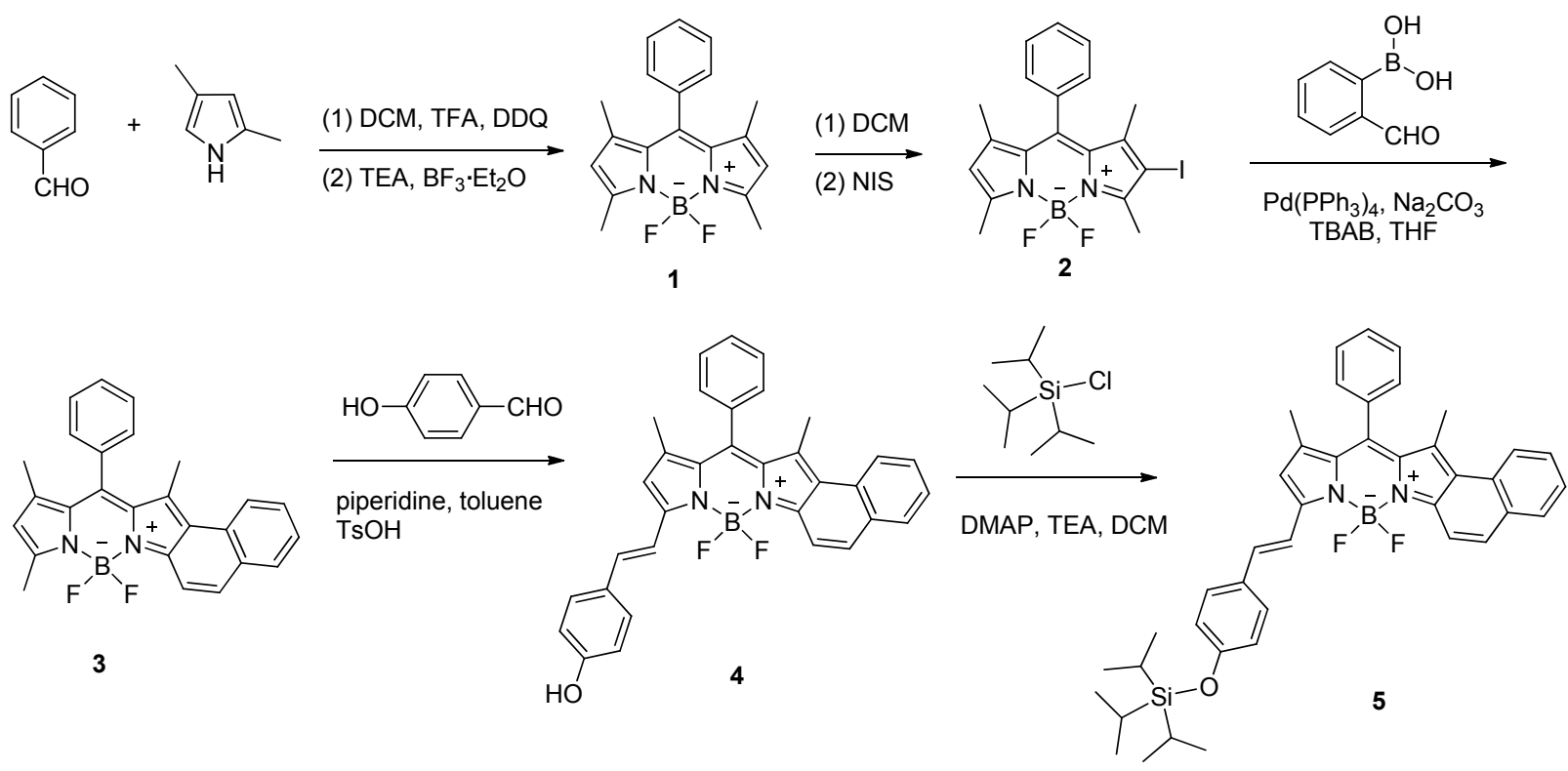

图式 1 探针分子 5 的合成路径

Scheme 1 Synthetic route of probe 5 

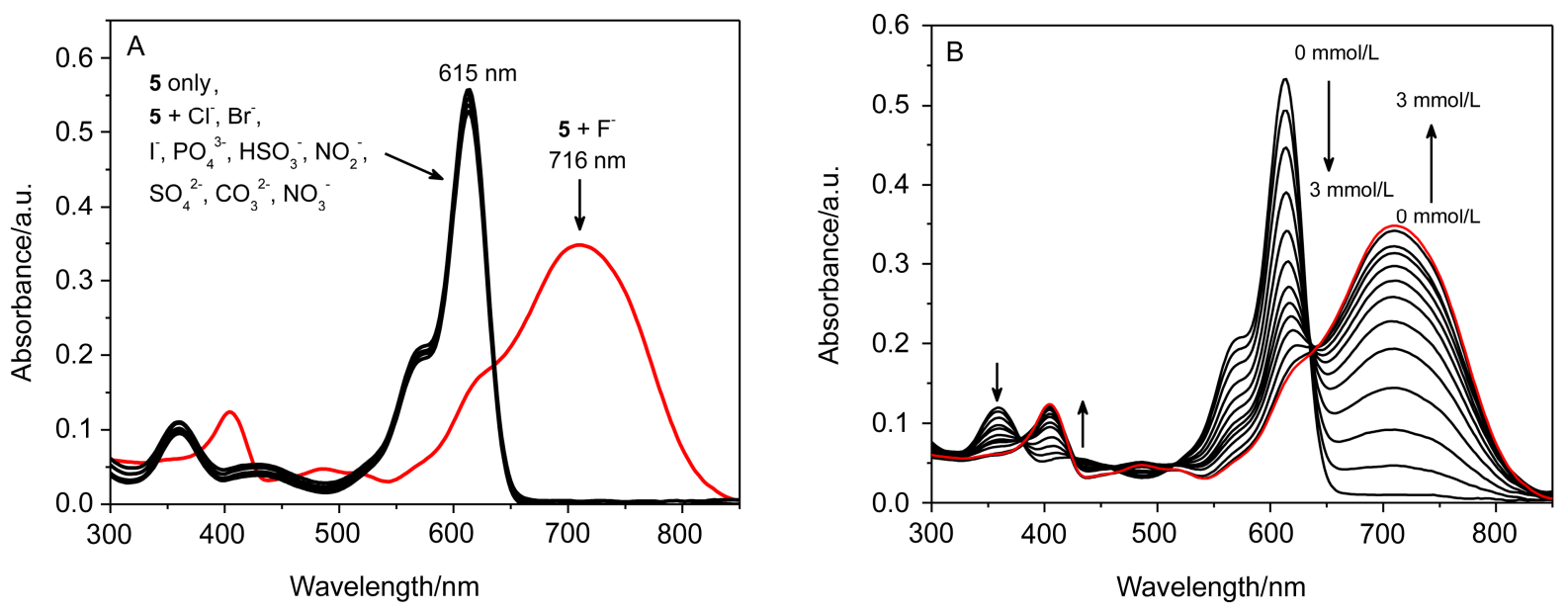

图 1 探针 $\mathbf{5}\left(10 \mu \mathrm{mol} \cdot \mathrm{L}^{-1}\right)$ 的乙腈溶液中加入 300 equiv. 阴离子溶液(A)和加入不同浓度 $\mathrm{TBAF}$ 溶液(B)之后的紫外-可见光谱图 Figure 1 Absorption spectra of probe $5\left(10 \mu \mathrm{mol} \cdot \mathrm{L}^{-1}\right)$ in $\mathrm{CH}_{3} \mathrm{CN}$ after the addition of 300 equiv. various anions (A) and various concentrations of TBAF (B)
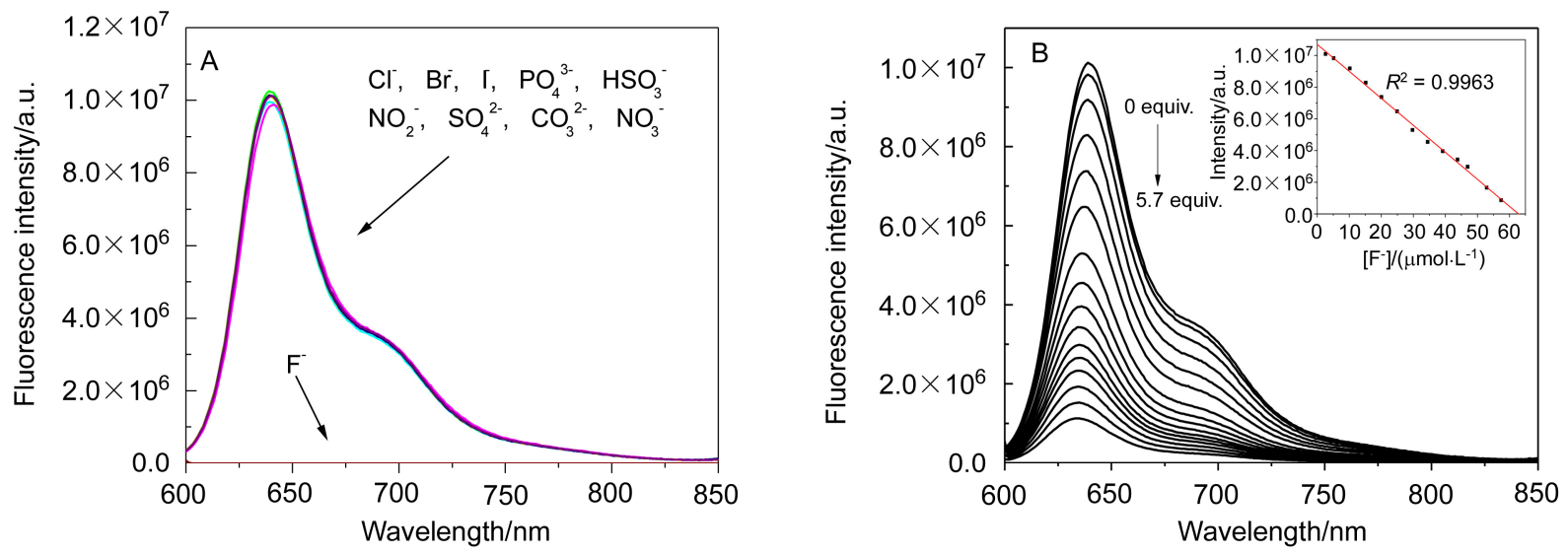

图 2 探针 $\mathbf{5}\left(10 \mu \mathrm{mol} \cdot \mathrm{L}^{-1}\right)$ 的乙腈溶液中加入 300 equiv. 不同阴离子溶液(A)和滴加不同浓度 TBAF 溶液(B)的荧光发射光谱(激发 波长为 $600 \mathrm{~nm})$

Figure 2 Fluorescence spectra of probe $5\left(10 \mu \mathrm{mol} \cdot \mathrm{L}^{-1}\right)$ in $\mathrm{CH}_{3} \mathrm{CN}$ after the addition of 300 equiv. various anions (A) and various concentrations of TBAF $\left(\lambda_{\mathrm{ex}}=600 \mathrm{~nm}\right)(\mathrm{B})$

The insert shows the linear relationship between the emission intensity of probe and concentrations of TBAF

其它阴离子时, 荧光光谱没有发生变化, 但是当 $\mathrm{F}^{-}$加入 时, 荧光的发射峰消失, 发生了荧光猝灭. 为了详细研 究苂光猝灭和离子浓度之间的定量关系, 我们做了不同 浓度 $\mathrm{F}^{-}$的滴定实验. 如图 2B 所示, 随着 $\mathrm{F}^{-}$浓度的增加, $648 \mathrm{~nm}$ 附近的荧光强度逐渐减低, 直至完全消失. 648 $\mathrm{nm}$ 处的苂光强度与 $\mathrm{F}^{-}$浓度呈现很好的线性关系, 线性 相关系数 $R^{2}=0.9963$ (图 2B 中的插图). 苂光对 $\mathrm{F}^{-}$浓度 的响应范围为 $0 \sim 63 \mu \mathrm{mol} \cdot \mathrm{L}^{-1}$. 这些测试结果表明, 探 针分子 5 对氟离子具有较高的选择性, 可作为氟离子的 比率计量型和近红外苂光 “turn off” 型探针.

\section{2 探针分子 5 对氟离子的检测机理研究}

由于氟和硅之间具有很强的亲和力, 氟离子的加入 很容易使探针分子 $\mathbf{5}$ 中的 $\mathrm{Si}-\mathrm{O}$ 键断裂, 产生去保护的<smiles>CC1=C2c3ccccc3C=C[N+]23C1=C(c1ccccc1)c1c(C)cc(/C=C/c2ccc(O[Si](C(C)C)(C(C)C)C(C)C)cc2)n1[B-]3(F)F</smiles>

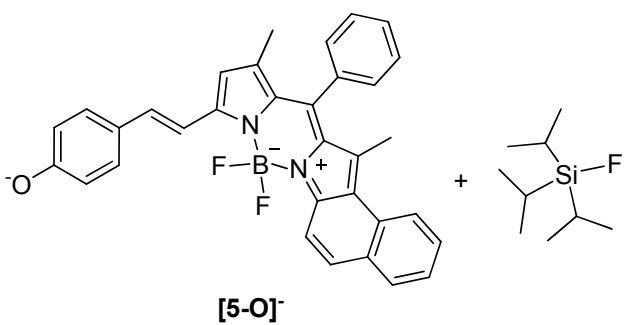
BODIPY 阴离子 [5-O $]^{-}$(Eq. 1), 高分辨质谱中发现 $m / z$ 
513.4632 的峰即归属于这一物质. 这种结构上的变化现 象与已发表文献中一致 ${ }^{[16,19]}$. [5-O ${ }^{-}$中的酚盐离子与 BODIPY 很好地共轭, 从而产生很强的分子内电荷转移 效应(ICT), 使苂光发生猝灭.

为了深入研究 $\mathrm{F}^{-}$对 BODIPY 探针结构和光谱性质 的影响, 我们利用 Gaussian 09 软件进行了理论计算. 首 先使用 B3LYP/6-31G(d)的函数和基组对探针分子 5 及 去保护的 [5-O $]^{-}$结构进行优化, 之后利用含时密度泛函 理论(TD-DFT)对这两个化合物的电子结构和光谱进行 了计算. 如图 3 所示, 两个化合物的吸收光谱主要来自 最高占据轨道(HOMO)到最低空轨道(LUMO)之间的跃 迁. 探针分子 5 的 HOMO 和 LUMO 主要离域在
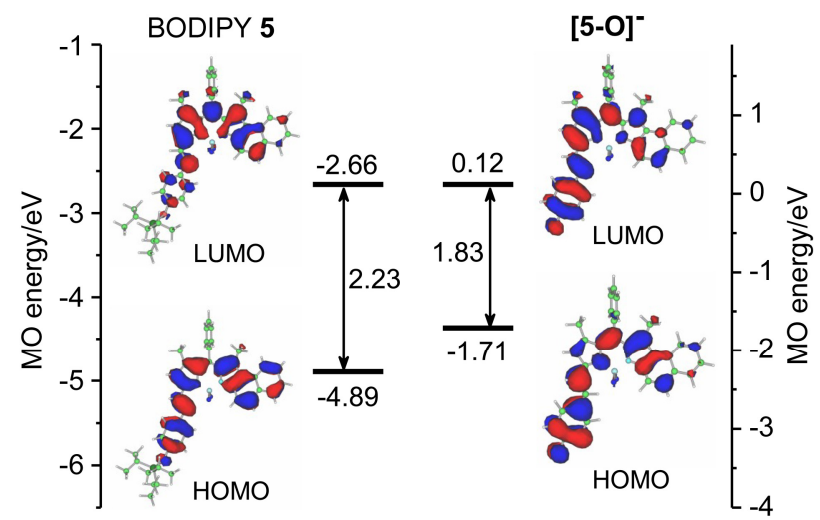

图 3 BODIPY 5 及去保护产物 $[5-0]^{-}$的前线分子轨道图及能 级图

Figure 3 Energy diagram of the frontier $\pi$-MOs of BODIPY 5 and $[\mathbf{5}-\mathbf{O}]^{-}$

The nodal patterns are shown at an isosurface value of 0.04 a.u. The MO energies for $[\mathbf{5}-\mathrm{O}]^{-}$are plotted against a secondary axis
BODIPY 母核上和 3-位取代苯环上，而失去硅烷之后的 [5-O $]^{-}$分子的 HOMO 和 LUMO 更多地离域到酚氧负离 子上. 因此，给电子的酚氧基团对 [5-O]一分子 $\mathrm{S} 1$ 激发 态的分子内电荷转移有重要贡献. 这可能就是探针分子 结合 $\mathrm{F}^{-}$之后产生荧光猝灭的原因 ${ }^{[9]}$. 探针分子 5 和去保 护的 [5-O $]^{-}$分子的 HOMO-LUMO 能级差分别为 2.23 和 $1.83 \mathrm{eV}, \mathrm{HOMO}-\mathrm{LUMO}$ 能级差的降低使紫外-可见光谱 红移, 这与实验得到的结果吻合.

\section{3 荧光探针在活体细胞中的成像研究}

我们使用活的 HeLa 细胞作为研究对象, 对探针 $\mathbf{5}$ 在活体细胞中检测 $\mathrm{F}^{-}$的性能进行了成像分析. 如图 4A 所示, $37{ }^{\circ} \mathrm{C}$ 下将 $\mathrm{HeLa}$ 细胞与 $10 \mu \mathrm{mol} \cdot \mathrm{L}^{-1}$ BODIPY 5 孵 育 $1 \mathrm{~h}$, 细胞表现出较强的红色荧光, 表明探针具有良 好的膜透性. 随后, 将 $\mathrm{F}^{-}$加入到细胞中培养 $1 \mathrm{~h}$, 用磷酸 盐缓冲溶液清洗, 除去多余的 $\mathrm{F}^{-}$. 如图 4B 所示, $\mathrm{F}^{-}$的加 入明显地猝灭了探针分子的荧光. 图 4C 和 4D 是使用特 异性荧光染料 Hoechst $33342^{[20]}$ 染色的细胞核的苂光. 由明场图 $4 \mathrm{E}$ 和 $4 \mathrm{~F}$ 可以看出, 在成像实验过程中细胞具 有较好的活性. 从图 $4 \mathrm{G}$ 融合图像可以看出, 探针 5 主要 聚集在细胞中细胞核周围的细胞质中，通过细胞核和细 胞质荧光的对比发现, 探针分子 5 可以很好地穿透细胞 膜进入细胞质，并用于活细胞中 $\mathrm{F}^{-}$的苂光检测.

\section{2 结论}

设计合成了一种新型不对称萘环稠合 BODIPY 探 针分子 5 , 通过质谱、氢谱对化合物的结构进行了表征. 探针分子 5 对 $\mathrm{F}^{-}$有特异性紫外和苂光响应. 由于 $\mathrm{F}^{-}$选
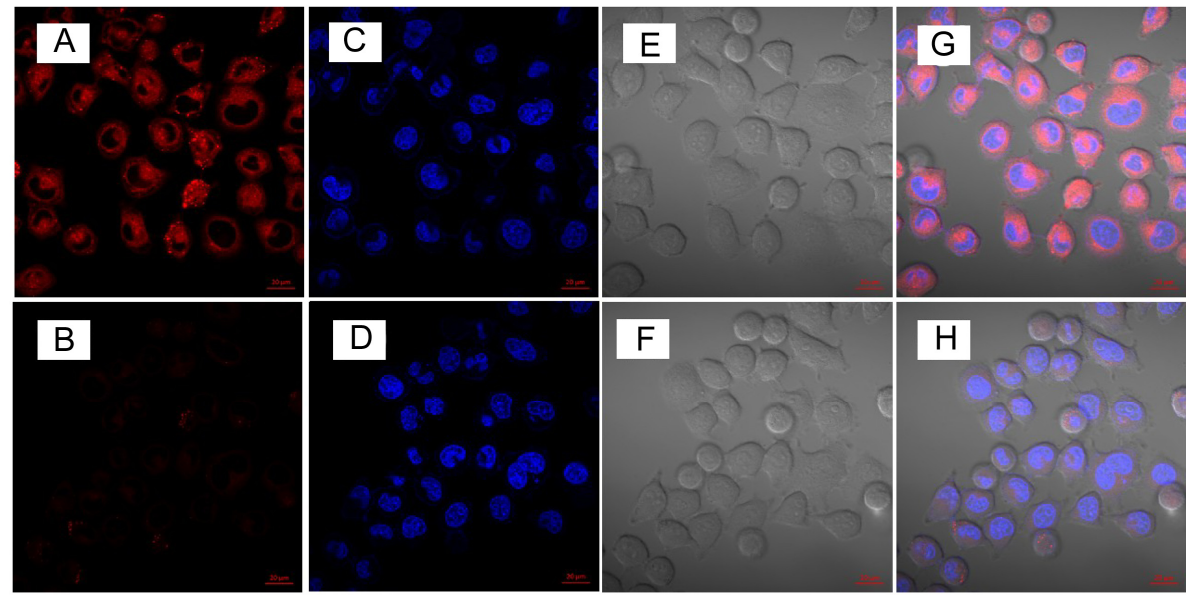

图 45 在 Hela 细胞中对氟离子的成像分析

Figure 4 Laser confocal micrographs of 5-incubated Hela cells

In the absence (A, C, E, G) and presence (B, D, F, H) of TBAF ( $\left.\lambda_{\mathrm{ex}}=600 \mathrm{~nm}\right)$. (A) and (B) show the intracellular fluorescence of $\mathbf{5}$, (C) and (D) show the fluorescence of cell nucleus stained by Hoechst 33342, (E) and (F) are the bright field images, $(\mathrm{G})$ and $(\mathrm{H})$ are the merged images. Hela cells were incubated with $10 \mu \mathrm{mol} \cdot \mathrm{L}^{-1} \mathbf{5}$ for $1 \mathrm{~h}$ at $37{ }^{\circ} \mathrm{C}$. For $\mathrm{F}^{-}$imaging, the $\mathbf{5}$-incubated Hela cells were subsequently incubated with $100 \mu \mathrm{mol} \cdot \mathrm{L}^{-1} \mathrm{TBAF}$ for $1 \mathrm{~h}$ at $37{ }^{\circ} \mathrm{C}$. 
择性地断裂 $\mathrm{Si}-\mathrm{O}$ 键, 在 $\mathrm{F}^{-}$存在时, 探针的紫外-可见 光谱红移 $100 \mathrm{~nm}$ 至近红外区域 $(716 \mathrm{~nm})$, 可用于肉眼比 色检测 F-. 而荧光则发生猝灭. TD-DFT 理论研究表 明, 荧光的猝灭可能是由分子内的电荷转移引起的. 细 胞成像研究也证实了在活细胞中探针分子 $\mathbf{5}$ 对氟离子具 有很好的专一选择性. 以上研究为生物体内检测 $\mathrm{F}^{-}$提 供了一种有效的方法.

\section{3 实验部分}

\section{1 仪器与试剂}

核磁共振氢谱和碳谱在 Bruker DRX400 核磁共振 仪上测试; 质谱在 Agilent 6110 HPLC-MS 上测试; 紫 外-可见光谱在 Shimadzu 3010 光谱仪上测试; 荧光光谱 在 Edinburgh FS5 光谱仪上测试, 石英比色㿼为 $1 \mathrm{~cm} \times 1$ $\mathrm{cm}$; 细胞成像实验所用荧光共聚焦显微镜为德国 Zeiss CLSM LSM700.

化合物 1 和 2 的合成方法参考已报道的文献[21], 化合物 3 的合成参见我们之前报道的文献[18]. 其它化 学试剂均购置于萨恩化学技术(上海)有限公司, 除非特 殊说明, 否则都是直接使用. 细胞核特异性荧光标记物 Hoechst 33342 购于 Invitrogen (Carlsbad, CA, USA). 所 用光谱测试用到的溶剂都是光谱纯试剂 (SigmaAldrich), $\mathrm{CH}_{2} \mathrm{Cl}_{2}$ 使用前用氢化钙回流重蒸.

\section{2 化合物 4 的合成}

在 $100 \mathrm{~mL}$ 茄型瓶中加入化合物 $3(30 \mathrm{mg}, 0.07$ $\mathrm{mmol}$ )、对羟基苯甲醛 $(11 \mathrm{mg}, 0.09 \mathrm{mmol}) 、$ 对甲苯磺酸 (1 mg, 催化量)、甲苯 $15 \mathrm{~mL}$ 和哌啶 $1 \mathrm{~mL}$. 安装 DeanStark 装置, 将混合溶液加热回流 $1 \mathrm{~h}$, 直至所用溶剂全 部蒸干, 用乙酸乙酯将粗产物溶解, 之后旋蒸除去溶剂, 乙酸乙酯/石油醚(体积比为 $7: 3$ ) 作洗脱剂, 用硅胶柱 分离得到 $20 \mathrm{mg}$ 黑色固体, 产率 53\%. m.p. 262 264 ${ }^{\circ} \mathrm{C}$; UV-Vis $\left(\mathrm{CH}_{2} \mathrm{Cl}_{2}\right) \lambda_{\text {max }}: 620 \mathrm{~nm} ;{ }^{1} \mathrm{H}$ NMR $(400 \mathrm{MHz}$, $\left.\mathrm{CDCl}_{3}\right) \delta: 8.25(\mathrm{~d}, J=8.2 \mathrm{~Hz}, 1 \mathrm{H}), 7.95(\mathrm{~d}, J=9.2 \mathrm{~Hz}$, $1 \mathrm{H}), 7.82(\mathrm{~d}, J=7.0 \mathrm{~Hz}, 1 \mathrm{H}), 7.69 \sim 7.75(\mathrm{~m}, 2 \mathrm{H}), 7.58$ (dd, $J=7.4,3.6 \mathrm{~Hz}, 5 \mathrm{H}), 7.32 \sim 7.51(\mathrm{~m}, 6 \mathrm{H}), 6.89$ (d, $J=$ $8.6 \mathrm{~Hz}, 2 \mathrm{H}), 6.75$ (s, 1H), 2.02 (s, 3H), $1.50(\mathrm{~s}, 3 \mathrm{H}) ;{ }^{13} \mathrm{C}$ NMR $\left(100 \mathrm{MHz}, \mathrm{CDCl}_{3}\right) \delta: 191.50,162.26,158.10$, 147.57, 146.10, 140.89, 140.37, 135.18, 132.56, 131.87, $130.48,129.99,129.72,129.45,129.30,128.76,127.04$, $124.72,124.34,124.07,123.83,120.23,118.96,116.09$, 34.90, 34.56, 31.93, 30.22, 29.37, 15.35, 14.92; ESI MS $m / z 514.06\left(\mathrm{M}^{+}\right)$. Anal. calcd for $\mathrm{C}_{33} \mathrm{H}_{25} \mathrm{BF}_{2} \mathrm{~N}_{2} \mathrm{O}: \mathrm{C}$ 77.06, $\mathrm{H}$ 4.90, N 5.45; found C 77.15, H 4.92, N 5.39.

\section{3 探针分子 5 的合成}

在 $100 \mathrm{~mL}$ 茄形瓶中加入化合物 $4(10 \mathrm{mg}, 0.02$ $\mathrm{mmol}$ )、4-二甲氨基吡啶(DMAP, $1 \mathrm{mg}, 0.008 \mathrm{mmol}$ ), 15 $\mathrm{mL}$ 新蒸 $\mathrm{CH}_{2} \mathrm{Cl}_{2}$. 室温搅拌溶解, 之后加入 3 滴三乙胺, 继续搅拌 $10 \mathrm{~min}$. 将三异丙基氯硅烷 $(0.02 \mathrm{~mL}, 0.1$ $\mathrm{mmol}$ )加入反应液中, 室温摚拌反应 $4 \mathrm{~h}$. 反应结束后加 入 $10 \mathrm{~mL}$ 蒸馏水, 然后用 $\mathrm{CH}_{2} \mathrm{Cl}_{2}$ 萃取, 无水硫酸钠干燥 得到有机相. 乙酸乙酯/石油醚(体积比 $1: 9$ ) 作洗脱剂, 硅胶柱层析提纯得到 $13 \mathrm{mg}$ 黑色固体, 产率 96\%. m.p. 248 $250{ }^{\circ} \mathrm{C}$; UV-Vis $\left(\mathrm{CH}_{2} \mathrm{Cl}_{2}\right) \lambda_{\text {max }}: 621 \mathrm{~nm} ;{ }^{1} \mathrm{H}$ NMR $\left(400 \mathrm{MHz}, \mathrm{CDCl}_{3}\right) \delta: 8.25(\mathrm{~d}, J=8.1 \mathrm{~Hz}, 1 \mathrm{H}), 7.95(\mathrm{~d}, J=$ $9.1 \mathrm{~Hz}, 1 \mathrm{H}), 7.84 \sim 7.80(\mathrm{~m}, 1 \mathrm{H}), 7.76 \sim 7.79(\mathrm{~m}, 2 \mathrm{H}), 7.57$ (dd, $J=5.8,2.9 \mathrm{~Hz}, 5 \mathrm{H}), 7.36 \sim 7.52(\mathrm{~m}, 5 \mathrm{H}), 6.92(\mathrm{~d}, J=$ $8.6 \mathrm{~Hz}, 2 \mathrm{H}), 6.75(\mathrm{~s}, 1 \mathrm{H}), 2.02$ (s, 3H), 1.50 (s, 3H), 1.29 $1.33(\mathrm{~m}, 3 \mathrm{H}), 1.12(\mathrm{~d}, J=7.2 \mathrm{~Hz} 18 \mathrm{H}) ;{ }^{13} \mathrm{C}$ NMR $(100$ $\left.\mathrm{MHz}, \mathrm{CDCl}_{3}\right) \delta: 158.24,157.84,147.71,147.64,147.13$, $145.89,145.51,140.91,140.06,138.58,138.49,136.93$, $135.36,133.56,133.29,131.76,130.53,130.07,129.75$, $129.71,129.28,129.21,128.82,126.94,124.50,124.27$, $124.22,124.01,123.84,120.50,120.14,119.13,116.80$, $116.28,37.13,34.90,34.55,31.96,30.23,17.93,15.36$, 14.93, 12.75; ESI MS $m / z$ : $670.18\left(\mathrm{M}^{+}\right)$. Anal. calcd for $\mathrm{C}_{42} \mathrm{H}_{45} \mathrm{BF}_{2} \mathrm{~N}_{2} \mathrm{OSi}$ : C 75.21, H 6.76, N 4.18; found C 75.32, H 6.80, N 4.22.

\section{4 理论计算}

密度泛函理论(DFT)构型优化使用高斯 G09W 软件 包 ${ }^{[22]}$ 进行, 选用 B3LYP 函数, 6-31 G(d,p) 基组, 电子结构 和光谱的计算采用 TD-DFT 方法.

\section{5 细胞成像实验}

将 HeLa 细胞接种于 $35 \mathrm{~mm}$ 共聚焦培养血中, 细胞 培养液组成为杜氏改良 Eagle 培养液(DMEM)加体积分 数为 $10 \%$ 胎牛血清, 并置于体积分数为 $5 \% \mathrm{CO}_{2} 、 37{ }^{\circ} \mathrm{C}$ 的二氧化碳培养箱中培养 $24 \mathrm{~h}$ 使细胞贴壁. 另外配制 $1.0 \mathrm{mmol} / \mathrm{L}$ BODIPY 5 的乙腈溶液, 并用磷酸盐缓冲溶 液 (PBS) 稀释至 $10 \mu \mathrm{mol} / \mathrm{L}$. 将贴壁后的细胞用 10 $\mu \mathrm{mol} / \mathrm{L}$ 探针溶液培养 $30 \mathrm{~min}$, 接着用 $0.01 \mathrm{~mol} / \mathrm{L}$ PBS 缓 冲液洗涤细胞三次, 用 Zeiss CLSM LSM700 共聚焦荧 光成像显微镜进行成像分析. 将 HeLa 细胞用 100 $\mu \mathrm{mol} / \mathrm{L} \mathrm{TBAF}$ 溶液孵育 $10 \mathrm{~min}$ 后加入 $10 \mu \mathrm{mol} / \mathrm{L}$ 探针孵 育 $30 \mathrm{~min}, \mathrm{PBS}$ 缓冲液洗涤后进行成像分析. 成像条件 为采用 $405 \mathrm{~nm}$ 激光器激发 Hoechst 33342, 发射波长收 集范围 420 到 $480 \mathrm{~nm} ; 633 \mathrm{~nm}$ 氦氖激光器激发 BODIPY 5, 发射波长收集范围为 645 到 $700 \mathrm{~nm}$. 
辅助材料(Supporting Information) BODIPY 4 和 5 的 核磁、高分辨质谱以及探针分子 $\mathbf{5}$ 在乙腈和酸、碱环境 中的紫外和荧光光谱图. 这些材料可以免费从本刊网站 (http://sioc-journal. cn/)上下载.

\section{References}

[1] (a) Kowada, T.; Maeda, H.; Kikuchi, K. Chem. Soc. Rev. 2015, 44, 4953.

(b) Zhu, H.; Fan, J.; Du, J.; Peng, X. Acc. Chem. Res. 2016, 49, 2115.

[2] Duke, R. M.; Veale, E. B.; Pfeffer, F. M.; Kruger, P. E.; Gunnlaugsson, T. Chem. Soc. Rev. 2010, 39, 3936.

[3] Bassin, E. B.; Wypij, D.; Davis, R. B.; Mittleman, M. A. Cancer, Causes Control, Pap. Symp. 2006, 17, 421.

[4] Wong, M. H.; Fung, K. F.; Carr, H. P. Toxicol. Lett. 2003, 137, 111.

[5] (a) Zhang, S. L.; Peng, X. J. J. Chem. Eng. 2016, 191 (in Chinese). (张世玲, 彭孝军, 化工学报, 2016, 191.)

(b) Zhang, H. M.; Wu, Y. C.; You, J. Y.; Cao, L.; Ding, S.; Jiang, K.; Wang, C. Y. Chin. J. Org. Chem. 2016, 36, 2559 (in Chinese). (张惠敏, 吴彦城, 尤嘉宜, 曹梁, 丁沙, 蒋凯, 汪朝阳, 有机化 学, 2016, 36, 2559.)

[6] Barbieri, A.; Bandini, E.; Monti, F.; Praveen, V. K.; Armaroli, N. Top. Curr. Chem. 2016, 374, 47.

[7] Fernandez, A.; Vendrell, M. Chem. Soc. Rev. 2016, 45, 1182.

[8] (a) Guo, Z.; Park, S.; Yoon, J.; Shin, I. Chem. Soc. Rev. 2014, 43, 16.

(b) Yuan, L.; Lin, W.; Zheng, K.; He, L.; Huang, W. Chem. Soc. Rev. 2013, 42, 622.

[9] Zou, B.; Liu, H.; Mack, J.; Wang, S.; Tian, J.; Lu, H.; Li, Z.; Shen, Z. RSC Adv. 2014, 4, 53864.

[10] (a) Kamkaew, A.; Lim, S. H.; Lee, H. B.; Kiew, L. V.; Chung, L. Y.; Burgess, K. Chem. Soc. Rev. 2013, 42, 77.

(b) Boens, N.; Leen, V.; Dehaen, W. Chem. Soc. Rev. 2012, 41, 1130 .

[11] (a) Lu, H.; Mack, J.; Yang, Y.; Shen, Z. Chem. Soc. Rev. 2014, 43, 4778.

(b) Lu, B. W.; Meng, S. X.; Feng, Y. Q. Chin. J. Org. Chem. 2018, 38,350 (in Chinese).

(卢博为, 孟舒献, 冯亚青, 有机化学, 2018, 38, 350.)

(c) Wang, S.; Liu, H.; Mack, J.; Tian, J.; Zou, B.; Lu, H.; Li, Z.; Jiang, J.; Shen, Z. Chem. Commun. 2015, 51, 13389.

(d) Wu, Q.; Wu, Y.; Yu, C.; Wang, Z.; Hao, E.; Jiao, L. Sens. Actuators, $B$ 2017, 253, 1079. (e) Ni, Y.; Wu, J. Org. Biomol. Chem. 2014, 12, 3774.

[12] (a) Ni, Y.; Zeng, W.; Huang, K.-W.; Wu, J. Chem. Commun. 2013, 49, 1217.

(b) Wakamiya, A.; Murakami, T.; Yamaguchi, S. Chem. Sci. 2013, 4, 1002.

[13] (a) Shen, Z.; Röhr, H.; Rurack, K.; Uno, H.; Spieles, M.; Schulz, B.; Reck, G.; Ono, N. Chem.-Eur. J. 2004, 10, 4853.

(b) Descalzo, A. B.; Xu, H.-J.; Xue, Z.-L.; Hoffmann, K.; Shen, Z.; Weller, M. G.; You, X.-Z.; Rurack, K. Org. Lett. 2008, 10, 1581.

[14] Umezawa, K.; Nakamura, Y.; Makino, H.; Citterio, D.; Suzuki, K. J. Am. Chem. Soc. 2008, 130, 1550.

[15] Wu, Y.; Cheng, C.; Jiao, L.; Yu, C.; Wang, S.; Wei, Y.; Mu, X.; Hao, E. Org. Lett. 2014, 16, 748 .

[16] Gai, L.; Mack, J.; Lu, H.; Nyokong, T.; Li, Z.; Kobayashi, N.; Shen, Z. Coord. Chem. Rev. 2015, 285, 24.

[17] (a) Sheng, W.; Zheng, Y.-Q.; Wu, Q.; Wu, Y.; Yu, C.; Jiao, L.; Hao, E.; Wang, J.-Y.; Pei, J. Org. Lett. 2017, 19, 2893.

(b) Sheng, W.; Cui, J.; Ruan, Z.; Yan, L.; Wu, Q.; Yu, C.; Wei, Y.; Hao, E.; Jiao, L. J. Org. Chem. 2017, 82, 10341.

(c) Zhou, X.; Wu, Q.; Feng, Y.; Yu, Y.; Yu, C.; Hao, E.; Wei, Y.; Mu, X.; Jiao, L. Chem.-Asian J. 2015, 10, 1979.

[18] Zhou, Z.; Zhou, J.; Gai, L.; Yuan, A.; Shen, Z. Chem. Commun. 2017, 53, 6621.

[19] Bozdemir, O. A.; Sozmen, F.; Buyukcakir, O.; Guliyev, R.; Cakmak, Y.; Akkaya, E. U. Org. Lett. 2010, 12, 1400.

[20] Huang, L.; Luo, Y.; Sun, X.; Ju, H.; Tian, J.; Yu, B.-Y. Biosens. Bioelectron. 2017, 92, 724.

[21] Lu, H.; Wang, Q.; Gai, L.; Li, Z.; Deng, Y.; Xiao, X.; Lai, G.; Shen, Z. Chem.-Eur. J. 2012, 18, 7852.

[22] Frisch, M. J.; Trucks, G. W.; Schlegel, H. B.; Scuseria, G. E.; Robb, M. A.; Cheeseman, J. R.; Scalmani, G.; Barone, V.; Mennucci, B.; Petersson, G. A.; Nakatsuji, H.; Caricato, M.; Li, X.; Hratchian, H. P.; Izmaylov, A. F.; Bloino, J.; Zheng, G.; Sonnenberg, J. L.; Hada, M.; Ehara, M.; Toyota, K.; Fukuda, R.; Hasegawa, J.; Ishida, M.; Nakajima, T.; Honda, Y.; Kitao, O.; Nakai, H.; Vreven, T.; Montgomery Jr., J. A.; Peralta, J. E.; Ogliaro, F.; Bearpark, M. J.; Heyd, J.; Brothers, E. N.; Kudin, K. N.; Staroverov, V. N.; Kobayashi, R.; Normand, J.; Raghavachari, K.; Rendell, A. P.; Burant, J. C.; Iyengar, S. S.; Tomasi, J.; Cossi, M.; Rega, N.; Millam, N. J.; Klene, M.; Knox, J. E.; Cross, J. B.; Bakken, V.; Adamo, C.; Jaramillo, J.; Gomperts, R.; Stratmann, R. E.; Yazyev, O.; Austin, A. J.; Cammi, R.; Pomelli, C.; Ochterski, J. W.; Martin, R. L.; Morokuma, K.; Zakrzewski, V. G.; Voth, G. A.; Salvador, P.; Dannenberg, J. J.; Dapprich, S.; Daniels, A. D.; Farkas, Ö.; Foresman, J. B.; Ortiz, J. V.; Cioslowski, J.; Fox, D. J. Gaussian 09, Revision B.01, Gaussian, Inc., Wallingford, CT, 2009. 